\title{
Automated width measurements of Martian dust devil tracks
}

\author{
Thiago Statella ${ }^{\mathrm{a}, *}$, Pedro Pina ${ }^{\mathrm{b}}$, Erivaldo Antônio da Silva ${ }^{\mathrm{c}}$ \\ ${ }^{a}$ Instituto Federal de Educação, Ciência e Tecnologia de Mato Grosso - IFMT, Brazil \\ ${ }^{\mathrm{b}}$ Centro de Recursos Naturais e Ambiente (CERENA), Instituto Superior Técnico - IST, Universidade de Lisboa, Av. Rovisco Pais, 1049-001 Lisboa, Portugal \\ ${ }^{\mathrm{C}}$ Universidade Estadual Paulista, Faculdade de Ciências e Tecnologia - FCT, 305 Roberto Simonsen, 19060-900 Presidente Prudente, Brazil
}

\section{A R T I C L E I N F O}

\section{Article history:}

Received 11 August 2014

Revised 19 September 2015

Accepted 3 November 2015

Available online 21 November 2015

\section{Keywords:}

Martian dust devil tracks

Mathematical Morphology

Aeolian process

\begin{abstract}
A B S T R A C T
Studying dust devils is important to better understand Mars climate and resurfacing phenomena. This paper presents an automated approach to calculate the width of tracks in orbital images. The method is based on Mathematical Morphology and was applied to a set of 200 HiRISE and MOC images of five Mars quadrangles, which were Aeolis, Argyre, Noachis, Hellas and Eridania. Information obtained by our method was compared with results of manual analysis performed by other authors. In addition, we show that track widths do not follow a normal distribution.
\end{abstract}

(C) 2015 Elsevier B.V. All rights reserved.

\section{Introduction}

Dust devils are whirlwinds made visible by entrained dust and sand. They are upward moving, spiraling flows caused by heating of near-surface air by insolation. The term dust devil is used to refer to sustained, particle-loaded convective vortices to distinguish them from vortices that form in the same way but are too weak to pick up materials and become visible (Balme and Greeley, 2006). They have been studied on Earth for more than a century (Baddeley, 1860; Brooks, 1960), were first observed on Mars in the 1970s in orbital images taken by the Viking orbiters (Thomas and Gierasch, 1985) and can achieve miles in width and height. The knowledge about dust devil activity contributes to the understanding of Martian climate, geology and surface modification which is essential to plan future robotic and manned missions (Atreya et al., 2006; Balme et al., 2003; Balme and Greeley, 2006; Ferri et al., 2003; Metzger et al., 1999; Rennó et al., 2000; Toigo et al., 2003). In addition, wind is the only surface-shaping factor that is known to presently be active over a short geological timescale on Mars (Örmo and Komatsu, 2003).

Information such as approximate width, orientation and area coverage about dust devils can be inferred from the analysis of their tracks (Hess, 2012). Such tracks are mostly dark filamentary features originated by differences in photometric properties of the surface, perhaps caused by removal of fine-grained material (Reiss et al., 2010).

* Corresponding author.

E-mail addresses: thiago.statella@cba.ifmt.edu.br (T. Statella), ppina@ist.utl.pt (P. Pina), erivaldo@fct.unesp.br (E.A. da Silva).
Most of the works regarding analysis of dust devils tracks in digital images use a manual method. There are hundreds of high resolution images depicting Martian surface being taken and the amount of information on them grew at a rate greater than the human capability to analyze and extract relevant information from these products to characterize the planet under study. As examples of the difficulty in analyzing manually so many images, Balme et al. (2003), Fisher et al. (2005), Cantor et al. (2006), Örmo and Komatsu (2003), Drake et al. (2006), and Whelley and Greeley (2006, 2008), had to search for tracks in (3000), (8116), (1238), (1700), (1734) and $(6002 ; 167,254)$ Mars Orbiter Camera (MOC) images, respectively; Stanzel et al. (2008) searched in (23) High Resolution Stereo Color (HRSC) images and Towner (2009) did that in (3079) Thermal Emission Imaging System (THEMIS) images.

We have previously proposed and evaluated automatic methods for detecting dust devil tracks in digital images (Statella et al., 2012), for inferring their direction of movement (Statella et al., 2014) and for estimating extensively the albedo contrast with their neighborhood (Statella et al., 2015). In this paper we present a method for calculating the mean width of tracks identified in Mars Global Surveyor (MGS) Mars Orbiter Camera (MOC) and Mars Reconnaissance Orbiter (MRO) High Resolution Imaging Science Experiment (HiRISE) images which is automated. Our approach is based on Mathematical Morphology.

\section{Image datasets}

A search for MOC narrow angle and HiRISE images with solar longitudes ranging between $180^{\circ}$ and $360^{\circ}$ (a few images out of 
Table 1

Summarized information about the image dataset.

\begin{tabular}{llrlcc}
\hline & Lat $^{\circ}$ & \multicolumn{1}{c}{ Lon $^{\circ}$} & \multicolumn{1}{l}{ Ls $^{\circ}$} & Width (pixels) & High (pixels) \\
\hline Min & -14.47 & 0.70 & 134.17 & 138.00 & 179.00 \\
Mean & -53.39 & 156.06 & 300.56 & 2754.85 & 2457.47 \\
Max & -64.80 & 358.80 & 353.90 & 9058.00 & 7526.00 \\
\hline
\end{tabular}

that range we knew beforehand containing tracks were also considered as part of the dataset) containing tracks of dust devils has been performed in the regions Aeolis, Noachis, Argyre, Eridania and Hellas.

More than a thousand images have been searched and a total of 124 images (75 MOC narrow angle panchromatic band and $49 \mathrm{HiR}-$ ISE red band) showing dark dust devil tracks were initially considered. In order to decrease the processing time (mainly because of the larger size of HiRISE images) and discard irrelevant information (like regions with no tracks) the images have been trimmed to its regions of interest (HiRISE images were also resampled by a factor of 1/3), making it a set of 200 images: 90 MOC and 110 HiRISE (a number of HiRISE scenes were trimmed into two or more regions of interest). After the trimming procedure, the final dataset was distributed as follows: $4 \mathrm{MOC}$ and 1 HiRISE images depicting regions in the Aeolis quadrangle, $20 \mathrm{MOC}$ and 19 HiRISE images depicting regions in the Noachis quadrangle, $16 \mathrm{MOC}$ and $55 \mathrm{HiR}-$ ISE images depicting regions in the Argyre quadrangle, $26 \mathrm{MOC}$ and 19 HiRISE images depicting regions in the Eridania quadrangle and $24 \mathrm{MOC}$ and 16 HiRISE images from the Hellas quadrangle. Table 1 summarizes some characteristics of the images. The spatial resolution of HiRISE images before resampling is either $0.25 \mathrm{~m} \mathrm{(} \sim 90 \%$ of the images) or $0.50 \mathrm{~m}$ while the MOC spatial resolution varies from $1.43 \mathrm{~m}$ to $8.75 \mathrm{~m}$ (mean $\sim 5 \mathrm{~m}$ ).
In Table $1, \mathrm{Ls}^{\circ}$ stands for solar longitude which refers to the Mars-Sun angle, measured from the Northern Hemisphere spring equinox where Ls $=0^{\circ}$. Fig. 1 shows the distribution of the initial set of 124 images (white circles) according to their center coordinates in the planetocentric system. The base map is the Mars Orbiter Laser Altimeter (MOLA) image layer of the Mars Global Digital Dune Database.

\section{Method}

The basic input for our method for calculating the width of tracks is a binary image containing the tracks. In such image, a dust devil track pixel is assigned to the value 1 (white) and all the background pixels are assigned to the value 0 (black). Therefore, a previous step in which dust devil tracks had been detected is needed. The tracks in each one of the 200 images had been automatically detected using the method proposed by Statella et al. (2012). It was the first automated method proposed for detecting dust devil tracks. The global accuracy in the detection was $92 \% \pm 5 \%$. A review of papers regarding the manual analysis of dust devil tracks is fully provided in Statella et al. (2012) as well as detailed information on the automated method developed by the authors. In Fig. 2 we show examples of the detection of tracks. Fig. 2(A) and (C) are original images HiRISE PSP_006163_1345 and MOC M10-01206, respectively. The HiRISE image has spatial resolution of $0.25 \mathrm{~m}$ and its size is $4415 \times 7184$ pixels. The MOC image has spatial resolution of $5.55 \mathrm{~m}$ and its size is $363 \times 829$ pixels. Both images are from the Argyre region. Fig. 2(B) and (D) are the results of the detection. They are examples of the binary images adopted as input for our method.

For measuring tracks width in digital images we have used a Mathematical Morphology tool called granulometric analysis.

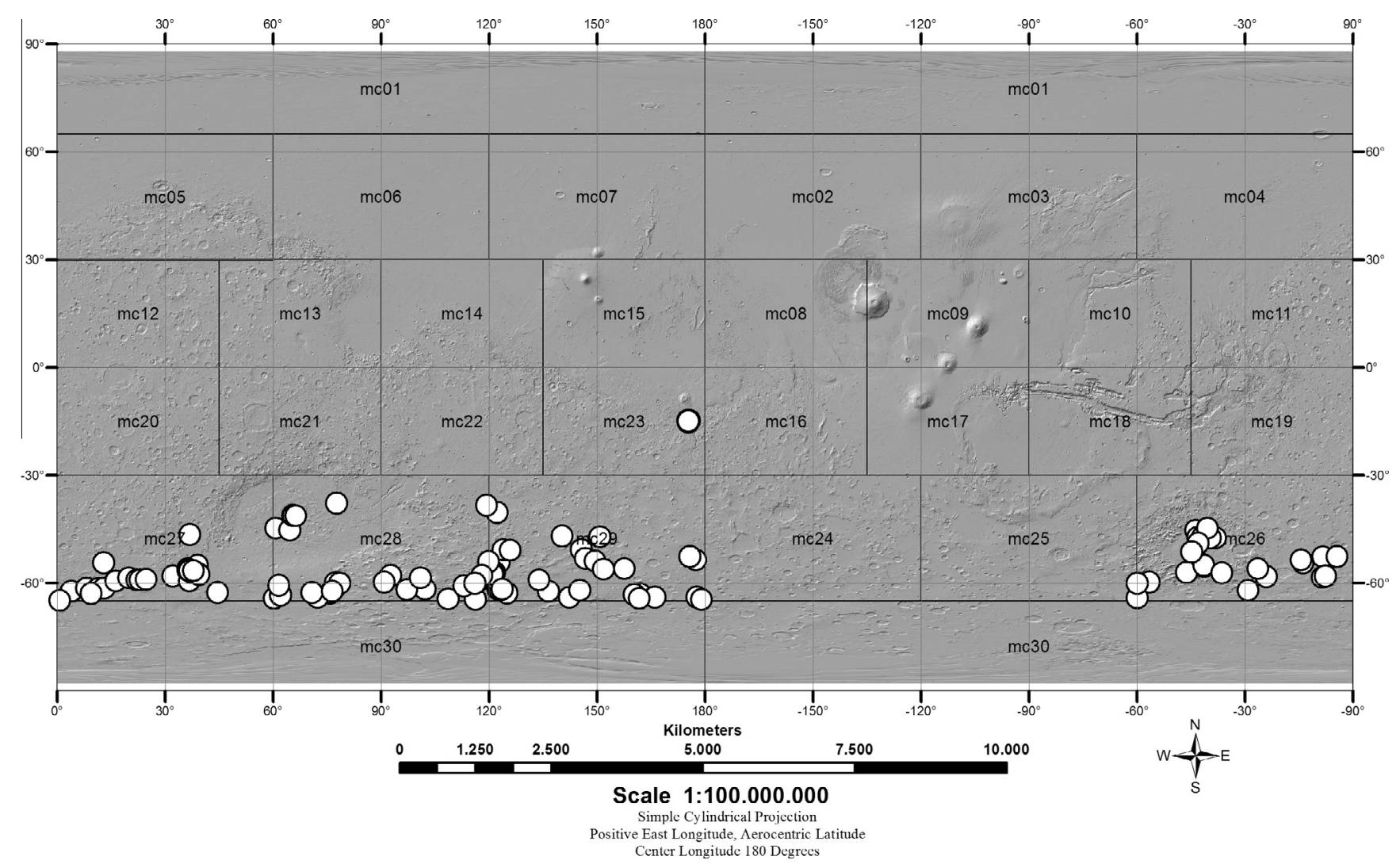

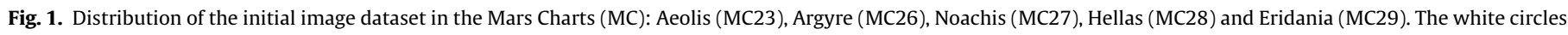
represent the center coordinates of the scenes. Image credits: R.K. Hayward, K.F. Mullins, L.K. Fenton, T.M. Hare, T.N. Titus, M.C. Bourke, A. Colaprete, P.R. Christensen. 

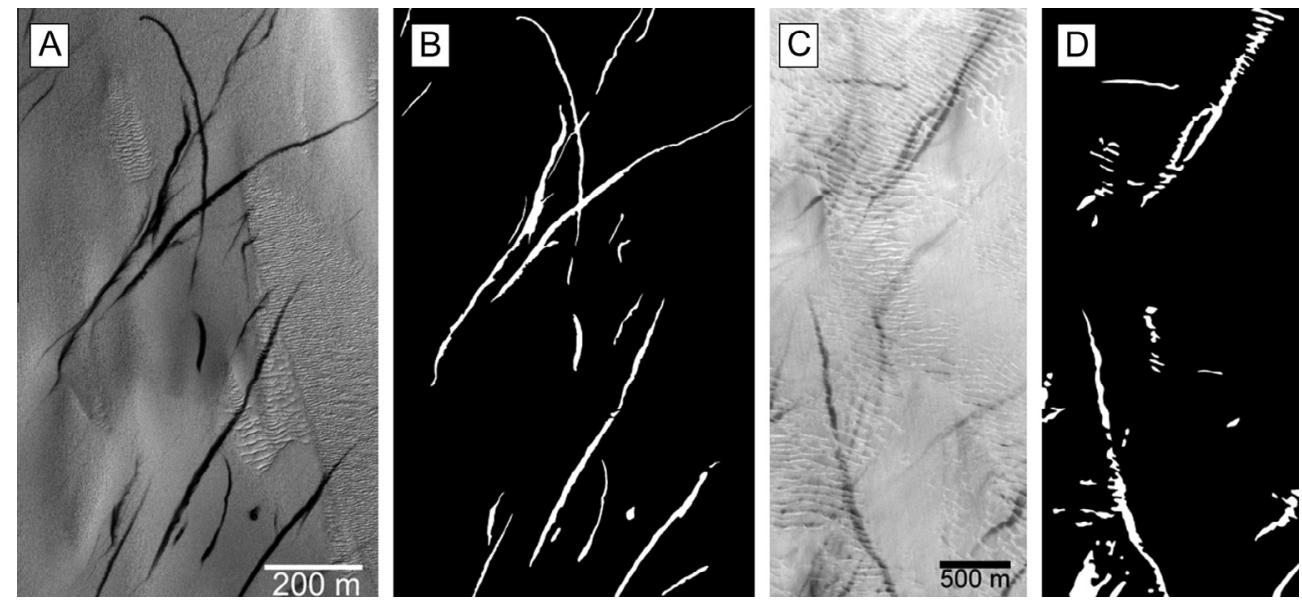

Fig. 2. Results of the automated tracks detection by the method proposed by Statella et al. (2012). (A) and (C): original HiRISE image PSP_006163_1345 and MOC image M1001206, both from the Argyre region. (B) and (D): the results of the automatic detection of the dust devil tracks in the original images.

Granulometric analysis gives information on the size distribution of the connected image features by applying successive morphological openings of increasing size (Serra, 1982). Morphological openings are based on morphological erosion and morphological dilation transforms, which can be defined as follows (Soille, 2004):

Let $B$ be a subset from $Z^{2}$ and $E$ a digital image, $B \subset E$. The erosion of an image $f$ by $B$ is the minimum of the translation of $f$ by the vectors $-b$ of $B$. $B$ is called structuring element (SE). Then we can write:

$\varepsilon_{B}(f)=\bigwedge_{b \in B} f_{-b}$

The dilation of $f$ by a SE B is the maximum of the translation of $f$ by the vectors $-b$ of $B$, so we write:

$\delta_{B}(f)=\bigvee_{b \in B} f_{-b}$.

The structuring element $B$ is a completely defined and known (size and shape) set which is compared, in a transformation, to the image unknown set. The result of this transformation allows us to evaluate the unknown set.

The morphological opening $\gamma$ of an image $f$ by $B$ is the erosion $\varepsilon$ of $f$ by $B$ followed by a dilation $\delta$ by $B$ transposed (or symmetric in relation to its origin) (Soille, 2004):

$\gamma_{B}(f)=\delta_{\breve{B}}\left[\varepsilon_{B}(f)\right]$.

In the granulometric analysis, the first opening operation filters out all image features that do not contain a SE of size one (in our case a digital equivalent of a disk with a diameter of 3 pixels). Then, a second opening operation by a disk of size 2 (diameter of 5 pixels) is applied and so forth. Formally, a granulometric analysis is defined as following: let $B \in Z^{2}$ be a SE. The family $\Gamma=\left(\gamma_{\lambda}\right)_{\lambda \geqslant 0}$ of openings by scales $\lambda B=\{\lambda b \mid b \in B\}, \lambda \geqslant 0, \lambda \in Z^{2}, B$ convex, is a granulometry. The concept of a granulometry may be linked to the sieving of rock or sediment grains in a gravel heap. The grains are sieved through screens of decreasing size mesh, leaving only the particles that are too big to pass through the sieve aperture. This is analogous to the opening of an image using a particular size of SE (Soille, 2004). The operation is performed pixel by pixel through the analysis of a neighborhood defined by the SE. The SE establishes the connectivity adopted in the erosion and dilation transforms. The erosion shrinks the subsets of the image and the dilation expands them. If a given subset is smaller than the SE adopted in the erosion step, then the subset cannot be recovered
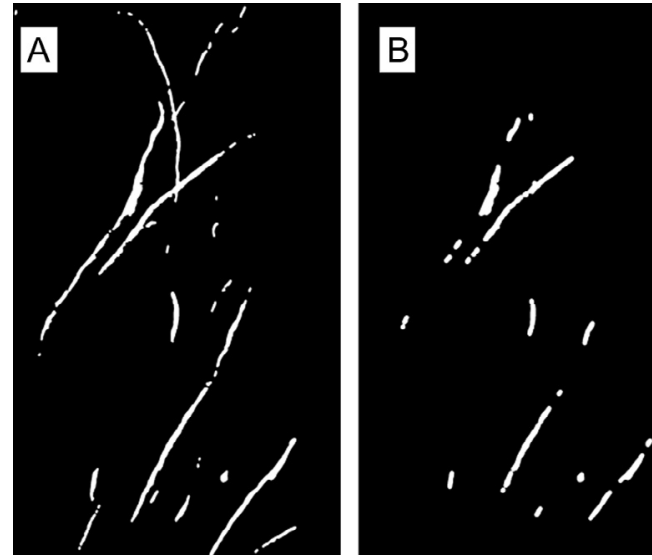

Fig. 3. Morphological opening transformations applied to the binary image PSP_006163_1345. (A) Opening by a 5 pixels radius disk; (B) opening by a 10 pixels radius disk.

by the following dilation. Therefore, after an opening, the remaining subsets in the image are all bigger than (or exactly fit) the SE adopted.

This procedure can be applied to infer the width distribution of dust devil tracks. As they are elongated features, a morphological opening with a disk SE larger (in radius) than the mean width of a track will filter most of the structure of that track and then can give us a measure of the mean width of that specific set or structure. In Fig. 3 we present the effect of opening transformations applied to the binary image PSP_006163_1345 shown in Fig. 2 (B). In Fig. 3(A) we show the image after being filtered by an opening by a disk with a radius of 5 pixels, and in Fig. 3(B) the result of the filtering with a disk SE of radius of 10 pixels is shown.

The amount of pixels removed by each opening can be plotted vs. the radius of the disk in the successive transformations in a graphic called pattern spectrum (Maragos, 1989). The pattern spectrum for the dust devil tracks of the image PSP_006163_1345 is shown in Fig. 4 and all relevant statistics can be extracted from it.

\section{Results and Discussion}

We have applied the method to the 200 HiRISE and MOC images from the Hellas, Eridania, Noachis, Argyre and Aeolis regions in our dataset. The images had been previously processed by Statella et al. 


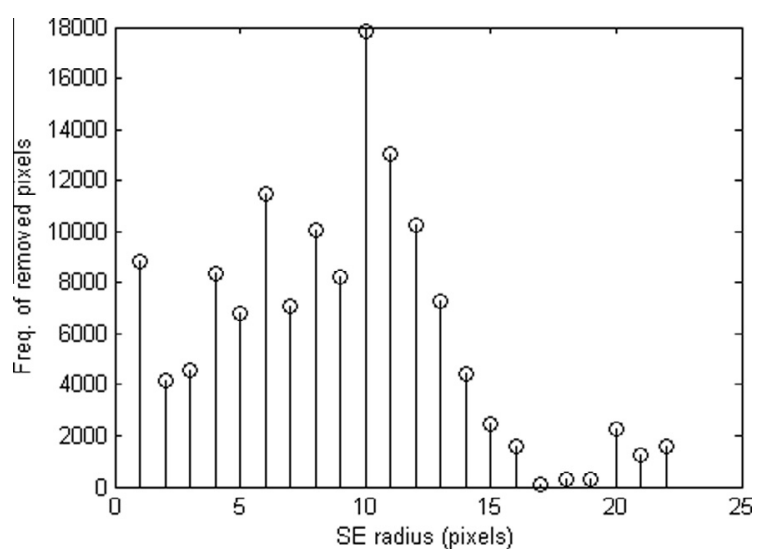

Fig. 4. Pattern spectrum of the HiRISE image PSP_006163_1345 shown in Fig. 2(B). Each bin in the above histogram represents the amount of pixels filtered out in successive morphological openings by structuring elements of increasing sizes (given by their radius).

Table 2

Dust devil tracks width in the study regions.

\begin{tabular}{lllll}
\hline Region & MTW $(\mathrm{m})$ & $\sigma(\mathrm{m})$ & MMTW $(\mathrm{m})$ & $\sigma(\mathrm{m})$ \\
\hline Aeolis & 43.58 & \pm 24.41 & 283.46 & \pm 114.05 \\
Noachis & 36.96 & \pm 26.31 & 159.93 & \pm 121.38 \\
Argyre & 28.83 & \pm 31.41 & 106.63 & \pm 113.21 \\
Eridania & 51.35 & \pm 60.98 & 208.41 & \pm 274.04 \\
Hellas & 64.24 & \pm 54.80 & 272.60 & \pm 218.33 \\
\hline
\end{tabular}

(2012) in order to detect automatically dust devil tracks (creation of binary images). The mean width (per image and per region) and the mean maximum width (per region) of the tracks created by dust devils are shown in Table 2. In that table, MTW and MMTW stand for Mean Track Width and Mean Maximum Track Width, respectively. And $\sigma$ stands for standard deviation. Verba et al. (2010) had performed a seasonal study on the Russell and Gusev craters, located in Noachis and Aeolis regions, respectively, using HiRISE images focusing dust devil tracks. According to them, tracks in Gusev are primarily formed by rare, large dust devils and smaller vortices fail to leave tracks that are visible from orbit, perhaps because of limited surface excavation depths.

On the other hand, they say that the Russell crater displays more frequent, smaller sinuous tracks than Gusev, which may be due to the thin dust cover in the Russell, allowing smaller dust devils to penetrate through the bright dust layer and leave tracks. Verba et al. (2010) had observed larger dust devils (track widths from 40 to $60 \mathrm{~m}$ ) on Gusev, whereas more numerous, smaller dust devils (widths from 30 to $40 \mathrm{~m}$ ) in the Russell crater. The dust devil tracks in the Russell crater are more numerous but shorter, more sinuous, and narrower than those in the Gusev crater. According to Table 2 our results agree with the cited authors. We have found a Mean Track Width of $43.58 \mathrm{~m} \pm 26.41 \mathrm{~m}$ for Aeolis, larger than the Mean Track Width of $36.96 \mathrm{~m} \pm 26.31 \mathrm{~m}$ we found for Noachis. The Mean Maximum Track Width was also larger for Aeolis $(283.46 \mathrm{~m} \pm 114.05 \mathrm{~m})$ than for Noachis $(159.93 \mathrm{~m} \pm 121.38 \mathrm{~m})$.

The large standard deviations are due to the fact that the dust devil tracks widths data do not follow a normal distribution. Rather, they are skewed to the right as can be seen in Figs. 5 and 6 that show, respectively, the MTW and MMTW histograms for the five regions.

In order to statically verify if those values of MTW and MMTW follow a normal distribution, we have applied the Anderson-Darling test to the data computed for Aeolis, Noachis, Argyre, Eridania and Hellas quadrangles. The null hypothesis, $H_{0}$ : data follow a normal distribution, has not been rejected only for Aeolis at a 5\% significance level. For the remaining regions, the null hypothesis has been rejected and therefore, data from Noachis, Argyre, Eridania and Hellas cannot be considered to follow a normal distribution.

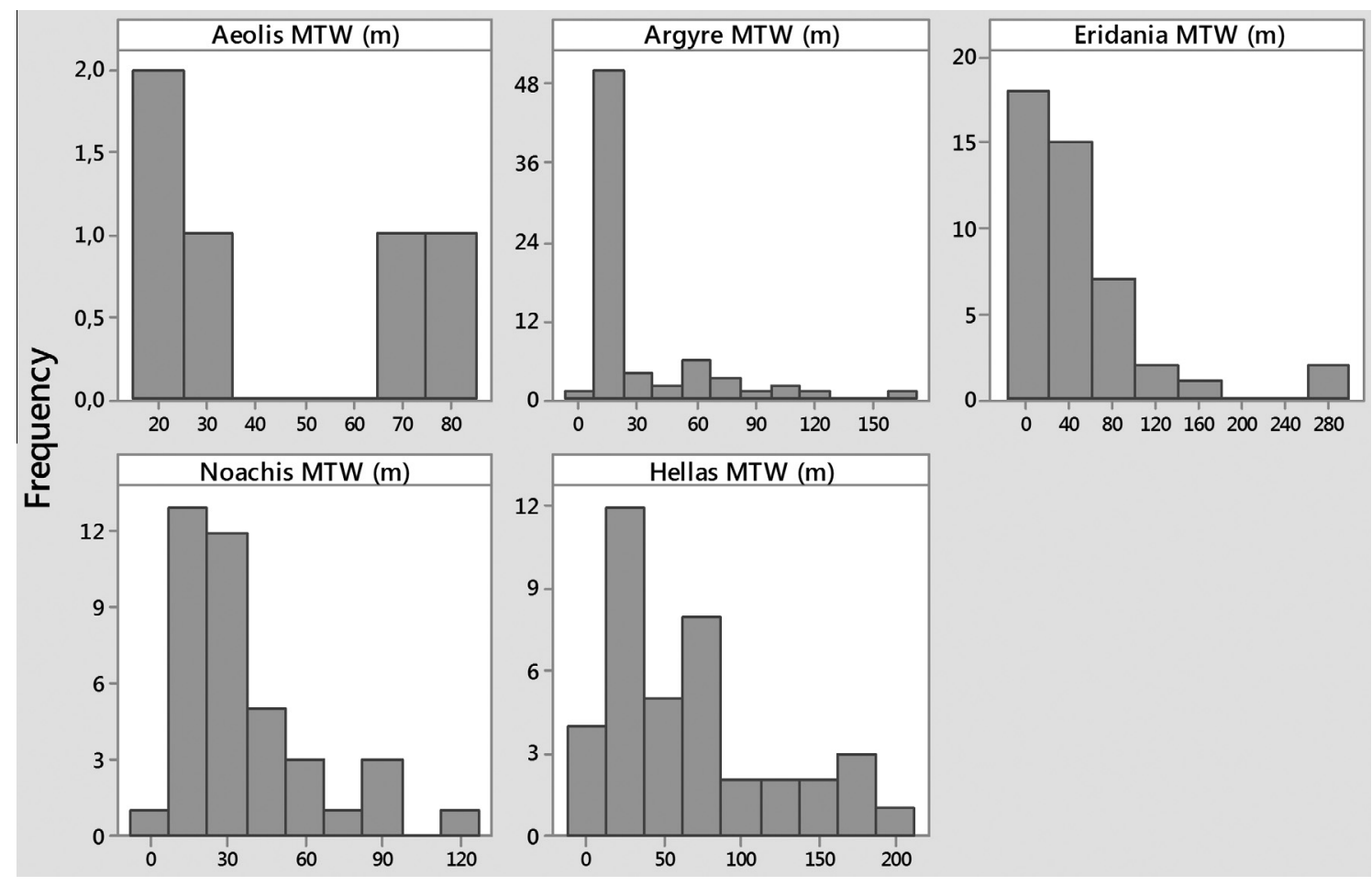

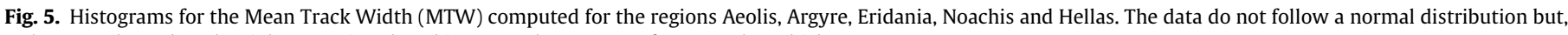
rather, are skewed to the right, meaning that thinner tracks are more frequent than thicker ones. 


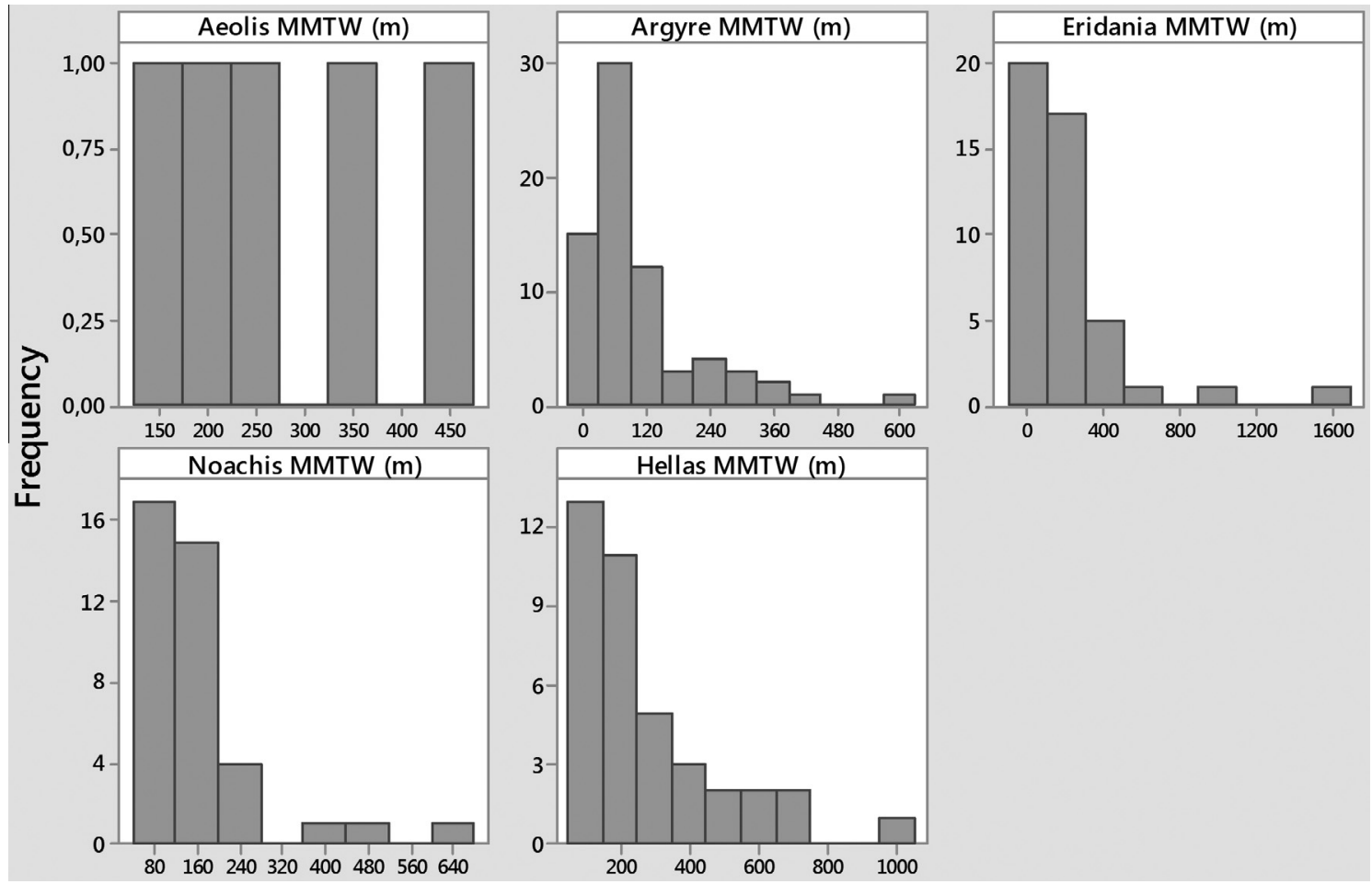

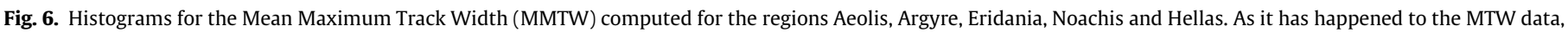
the MMTW data are also skewed to the right.

However, the exception of Aeolis should not be considered relevant since the number of processed images belonging to that region is too small. By comparison with the results from other quadrangles, we have reasons to believe that had the number of images for Aeolis been bigger, the skew to the right would have occurred in a similar way. Nevertheless, the skewness to the right is a clear indicator that thinner tracks are more frequent than thicker ones in all regions.

Next, we have applied the Mann-Whitney test (for non-normal data) to paired regions so that we could find out if there was any statistically significant difference between them. The tested pairs were Argyre vs. Eridania, Argyre vs. Noachis, Argyre vs. Hellas, Eridania vs. Noachis, Eridania vs. Hellas and Noachis vs. Hellas. Regarding the MTW data we have found that Argyre vs. Eridania, Argyre vs. Noachis, Argyre vs. Hellas can be considered different at a $5 \%$ significance level. On the other hand, there is no reason to believe that Eridania vs. Noachis, Eridania vs. Hellas and Noachis vs. Hellas MTW data are different. When it comes to the MMTW data we have found the following results: Argyre vs. Eridania, Argyre vs. Noachis, Argyre vs. Hellas, Eridania vs. Hellas and Noachis vs. Hellas can be considered different at a 5\% significance level, whilst Eridania vs. Noachis cannot.

\section{Summary and Conclusions}

Studying Martian dust devils is important to provide a better understanding of aeolian processes, low atmospheric behavior and resurfacing, among others. In this paper we have applied an automatic method for calculating mean and mean maximum dust devil track width in MOC and HiRISE images. The method is based on Mathematical Morphology and uses granulometric analysis to infer the tracks width. It is suitable to be applied to binary images containing tracks as input. The input images for the experiments had been obtained by Statella et al. (2012). Track widths have been calculated for 200 images from Martian regions of Aeolis, Eridania,
Hellas, Noachis and Argyre. Our results, obtained by an automatic method, agree to a manual analysis performed by Verba et al. (2010) in the Russell and Gusev craters, located in the regions Aeolis and Noachis, respectively. In addition, we have shown that the MTW and MMTW data do not follow a normal distribution. They show a skew to the right, meaning that thinner tracks (and therefore vortices with smaller diameters) are more frequent than thicker (vortices with bigger diameters) ones. In future work we will span the application of this approach to other regions of Mars.

\section{References}

Atreya, S.K., Wong, A., Rennó, N.O., Farrel, W.M., Delory, G.T., Sentman, D.D., Cummer, S.A., Marshall, J.R., Rafkin, S.C.R., Catling, D.C., 2006. Oxidant enhancement in Martian dust devils and storms: implications for life and habitability. Astrobiology 6, 439-450.

Baddeley, P.F.H., 1860. Whirlwinds and Dust Storms of India. Bell and Daldy, London.

Balme, M.R., Greeley, R., 2006. Dust devils on Earth and Mars. Rev. Geophys. 44, RG3003.

Balme, M.R., Whelley, P.L., Greeley, R., 2003. Mars: dust devil track survey in Argyre Planitia and Hellas Basin. J. Geophys. Res. Planets 108 (E8), 5086.

Brooks, H.B., 1960. Rotation of dust devils. J. Meteorol. 17, 84-86.

Cantor, B.A., Kanak, K.M., Edgett, K.S., 2006. Mars orbiter camera observations of Martian dust devils and their tracks (September 1997-January 2006) and evaluation of theoretical vortex models. J. Geophys. Res. Planets 111, E12002.

Drake, N.B., Tamppari, L.K., Baker, R.D., Cantor, B.A., Hale, A.S., 2006. Dust devil tracks and wind streaks in the North Polar region of Mars: a study of the 2007 phoenix Mars lander sites. Geophys. Res. Lett. 33 (19), L19S02.

Ferri, F., Smith, P.H., Lemmon, M., Rennó, N.O., 2003. Dust devils as observed by Mars pathfinder. J. Geophys. Res. Planets 108 (E12), 5133.

Fisher, J.A., Richardson, M.I., Newman, C.E., Szwast, M.A., Graf, C., Basu, S., Ewald, S. P., Toigo, A.D., Wilson, R.J., 2005. A survey of Martian dust devil activity using Mars global surveyor Mars orbiter camera images. J. Geophys. Res. Planets 110, E03004.

Hess, R., 2012. Short-lived and long-lived dust devil tracks in the coastal desert of Southern Peru. Aeolian Res. 5, 101-106.

Maragos, P., 1989. Pattern spectrum and multiscale shape representation. IEEE Trans. Pattern Anal. Mach. Intell. 11 (7), 701-716.

Metzger, S.M., Carr, J.R., Johnson, J.R., Parker, T.J., Lemmon, M.T., 1999. Dust devil vortices seen by the Mars pathfinder camera. Geophys. Res. Lett. 26 (18), 2781 2784. 
Örmo, J., Komatsu, G., 2003. Mars orbiter camera observation of linear and curvilinear features in the Hellas Basin: indications for multiple processes of formation. J. Geophys. Res. Planets 108 (E6), 5059.

Reiss, D., Raack, J., Rossi, A.P., Di Achille, G., Hiesinger, H., 2010. First in-situ analysis of dust devil tracks on Earth and their comparison with tracks on Mars. Geophys. Res. Lett. 37, L14203.

Rennó, N.O., Nash, A.A., Lunine, J., Murphy, J., 2000. Martian and terrestrial dust devils: test of a scaling theory using pathfinder data. J. Geophys. Res. Planets 105 (E1), 1859-1865.

Serra, J., 1982. Image Analysis and Mathematical Morphology. Academic Press, London.

Soille, P., 2004. Morphological Image Analysis. Springer-Verlag, Berlin.

Stanzel, C., Patzold, M., Williams, D.A., Whelley, P.L., Greeley, R., Neukum, G., 2008. Dust devils speeds, directions of motion and general characteristics observed by the Mars express high resolution stereo camera. Icarus 197, 39-51.

Statella, T., Pina, P., Silva, E.A., 2012. Image processing algorithm for the identification of Martian dust devil tracks in MOC and HiRISE images. Planet. Space Sci. 70, 46-58.
Statella, T., Pina, P., Silva, E.A., 2014. Automated determination of the orientation of dust devil tracks in Mars orbiter images. Adv. Space Res. 53, 1822-1833.

Statella, T., Pina, P., Silva, E.A., 2015. Extensive computation of albedo contrast between Martian dust devil tracks and their neighboring regions. Icarus 250 43-52.

Thomas, P., Gierasch, P.J., 1985. Dust devils on Mars. Science 230, 175-177.

Toigo, A.D., Richardson, M.I., Ewald, S.P., Gierasch, P.J., 2003. Numerical simulation of Martian dust devils. J. Geophys. Res. Planets 108 (E6), 5047.

Towner, M.C., 2009. Characteristics of large Martian dust devils using Mars odyssey thermal emission imaging system visual and infrared images. J. Geophys. Res. Planets 114, E02010.

Verba, C.A. Geissler, P.E. Titus, T.N., Waller, D., 2010. Observations from the high resolution imaging science experiment (HiRISE): Martian dust devils in Gusev and Russell craters. J. Geophys. Res. Planets 115, E09002.

Whelley, P.L., Greeley, R., 2006. Latitudinal dependency in dust devil activity on Mars. J. Geophys. Res. Planets 111, E10003.

Whelley, P.L., Greeley, R., 2008. The distribution of dust devil activity on Mars. J Geophys. Res. Planets 113, E07002. 